\section{The Effect of Deficit Irrigation and Crop Load on Leaf and Fruit Nutrition of Fertigated 'Ambrosia'/'M.9' Apple}

\author{
Gerry H. Neilsen ${ }^{1}$ and Denise Neilsen \\ Pacific Agri-Food Research Centre, Agriculture and Agri-Food Canada, \\ Summerland, BC, Canada VOH $1 Z 0$ \\ Sung-hee Guak \\ Chonbuk National University, Department of Horticulture, Jeonju, 561-256, \\ Korea
}

\section{Tom Forge \\ Pacific Agri-Food Research Centre, Agriculture and Agri-Food Canada, Summerland, BC, Canada VOH $1 Z 0$}

Additional index words. leaf and fruit N, P, K, Ca, Mg, and B, Malus $\times$ sylvestris Mill. var. domestica (Borkh.) Mansf.

\begin{abstract}
Mature, fruiting 'Ambrosia'/'M.9' apple [Malus $\times$ sylvestris (L.) Mill. var. domestica (Borkh.) Mansf.] trees were subjected over three growing seasons to a splitplot experimental design involving four irrigation main plot treatments and three subplot crop load treatments with six replicates. This semiarid production region is traditionally irrigated 01 May to 01 Oct. during which time an average of $\approx 15 \mathrm{~cm}$ of precipitation occurs. Irrigation treatments were applied through $2 \times 4 \mathrm{~L} \cdot \mathrm{h}^{-1}$ emitters per tree and included I1: daily application of 100\% evapotranspiration (ET); or I2: 50\% daily ET; or I3: $50 \%$ ET applied to one side; and I4: 50\%, 25\%, or $18 \%$ ET-application, applied every second day, 2007-09, respectively. Crop load treatments were imposed annually $\approx 4$ to 5 weeks after full bloom to create low $\left(2.5,3\right.$, and 3.75 fruits $/ \mathrm{cm}^{2}$ trunk cross-sectional area (TCSA), medium (4.5, 6, and 7.5 fruits $/ \mathrm{cm}^{2}$ TCSA), and high crop loads $(9,12$, and 15 fruits $/ \mathrm{cm}^{2}$ TCSA), 2007-09, respectively. Leaf and fruit nutrient concentration was affected more by crop load than by any deficit irrigation strategy. Increased crop load increased concentrations of leaf nitrogen $(N)$, calcium $(\mathrm{Ca})$, and fruit $\mathrm{Ca}$ in 2 of 3 years and consistently decreased concentrations of leaf and fruit phosphorus $(P)$ and potassium $(\mathrm{K})$ and, in 2 of 3 years, fruit boron (B). Reductions in seasonal water applications (as with I4) reduced leaf $P$ in 2 of 3 years. But, when significant, (usually only 1 of 3 year) increased fruit $\mathrm{Ca}$, magnesium (Mg), $\mathrm{P}, \mathrm{K}$, and $\mathrm{B}$ concentrations. Crop load also had a dominant effect on fruit nutrient removal rates expressed as kilograms per hectare. High crop load increased removal of all measured nutrients in most years. In contrast, imposition of deficit irrigation strategies often ( 2 of 3 years) reduced fruit $\mathrm{P}, \mathrm{Mg}$, and $\mathrm{B}$ removal rates but had little effect on $\mathrm{N}$, Ca, and $\mathrm{K}$. Cumulative evidence suggests that deficit irrigation applied to $\mathrm{N}, \mathrm{P}, \mathrm{K}$, and $\mathrm{B}$ fertigated high density 'Ambrosia' apple orchards in combination with crop load reduction to maintain fruit size should usually not create additional nutrient problems. However, low fruit Ca concentrations may occur if the crop is very low. Fertigation of $20 \mathrm{~g} \mathrm{~K} /$ tree/year was insufficient for older trees because inadequate $K$ occurred in all treatments by the third year.
\end{abstract}

Concern has been expressed about the sustainability of irrigation water supply in western North America in response to the role of climate change in reducing groundwater supply and winter mountain snowpack

Received for publication 16 Apr. 2015. Accepted for publication 8 July 2015 .

This work was primarily supported by Agriculture and Agri-Food Canada A-base funding. We thank Linda Herbert for chemical analyses, Istvan Losso for fertigation and irrigation applications and Bill Rabie for horticultural maintenance. Several University co-op students assisted with annual sampling and crop harvest.

${ }^{1}$ Corresponding author. E-mail: gerry.neilsen@, agr.gc.ca.
(Barnett et al., 2004; Payne et al., 2004). The problem is particularly important in the semiarid fruit growing region of the southern interior of British Columbia, where agriculture is an important economic activity and vulnerable to water restrictions resulting from conflicts with domestic water requirements (Neilsen et al., 2006).

Adaptive strategies to cope with insufficient water to fulfill irrigation requirements of fruit trees have previously been reported from nearby Washington State (Proebsting and Middelton, 1980; Proebsting et al., 1981). Regulated deficit irrigation has more recently been proposed to minimize water use, control vegetative growth, and maintain or even enhance apple fruit quality (Mills et al., 1996; Mpelasoka et al., 2001; Fallahi et al.,
2011). Such techniques may require management of crop load to maintain fruit size (Naschitz and Naor, 2005; Wünsche et al., 2000). In the Okanagan region, crop load reductions were used over a single growing season to maintain fruit size under drought conditions (Neilsen et al., 2010).

The nutritional implications of reducing water applications to previously wellwatered and fertilized deciduous trees have been little studied (Behboudian and Mills, 1997). Deficit irrigation treatments reduced 'Braeburn' apple $\mathrm{N}$ and $\mathrm{Ca}$ concentrations (Mills et al., 1996) but increased 'Anjou' pear fruit Ca concentrations (Brun et al., 1985). It has, however, been generally concluded that deficit irrigation has limited effects on fruit mineral nutrition which, if affected, is generally modified to improve fruit quality (Behboudian and Mills, 1997). In contrast, crop load has long been recognized as a major influence on apple leaf and fruit nutrient concentration (Hansen, 1973, 1980). Heavy cropping often stimulates tree $\mathrm{K}$ uptake, resulting in a depression of leaf $K$ relative to fruit $\mathrm{K}$ concentration (Jadczuk and Lenz, 1998). Changes in fruit nutrient concentrations can alter fruit quality as demonstrated by Volz et al. (1993), who found that lightly cropped trees produced fruit with reduced concentrations of $\mathrm{Ca}$ relative to $\mathrm{K}$ and a greater susceptibility to Ca-related disorders. Heavily cropped trees produced fruit with higher $\mathrm{Ca}$ concentrations relative to lightly cropped trees, irrespective of fruit size (Ferguson and Watkins, 1992). Despite several studies indicating that apple fruit size is strongly affected by an interaction between irrigation and crop load (Naor et al., 1997; Naschitz and Naor, 2005), there is limited information concerning how such an interaction would affect fruit mineral concentration and fruit quality at harvest.

This study was designed to test whether reducing crop load in response to deficit irrigation has negative consequences for leaf and fruit nutrition of fertigated apple trees.

\section{Materials and Methods}

'Ambrosia' on 'M.9' apple trees [Malus $\times$ sylvestris (L.) Mill. var. domestica (Borkh.) Mansf.] were planted in 2003 in a 0.9 (within row) $\times 3.5 \mathrm{~m}$ (between row) spacing on a Skaha loamy sand soil (Wittneben, 1986) in Summerland, British Columbia, Canada. During the experimental period, from 2007 to 2009 , the apple trees were in full fruit production and had filled their allotted space. By the end of the experiment, average TCSA was $13.7 \mathrm{~cm}^{2}$ and tree height $\approx 2 \mathrm{~m}$. Soil at the experimental site is an Aridic Haploxeroll, extensively planted to orchards or vineyards in the region. The region is a major Canadian fruit growing area characterized by cool winters (average temperature $1.4^{\circ} \mathrm{C}$, December to February) and warm summers (average temperature $19.4{ }^{\circ} \mathrm{C}$, June to August) requiring irrigation during the growing season. From 1 May to 1 Oct., reference evapotranspiration $\left(\mathrm{ET}_{\mathrm{o}}\right)$ values average $70 \mathrm{~cm}$ and 
annual precipitation $15 \mathrm{~cm}$. The trees were established and maintained for the first four growing seasons under a fully automated drip irrigation system comprising two $4-\mathrm{L} \cdot \mathrm{h}^{-1}$ pressure-compensating emitters placed $0.3 \mathrm{~m}$ on either side of the tree in their respective rows resulting in two emitters per tree. Irrigation quantity was applied daily in response to the previous day's evapotranspiration demand measured by an electronic atmometer (ET Gauge Co., Loveland, CO) (Parchomchuk et al., 1996). Atmometer readings were converted to plant water demand by taking into account the overestimation of Penman-Monteith $\mathrm{ET}_{\mathrm{o}}$ by atmometer reading as (actual $\mathrm{ET}_{\mathrm{a}}=0.75 \mathrm{ET}_{\mathrm{o}}$ ) and using a seasonal crop coefficient curve for apple (Neilsen et al., 2015) derived from Allen et al. (1998). To ensure the trees were not nutrient limited, and following optimum industry recommendations, trees received annual NPKB fertigation. $\mathrm{N}$ was fertigated daily as calcium nitrate $(15.5 \mathrm{~N}-0 \mathrm{P}-0 \mathrm{~K})$ for 6 weeks after bloom to provide $75 \mathrm{~g} \mathrm{~N} /$ tree. Subsequent research indicated that the $\mathrm{N}$ application rates were in excess of requirements for high-density apple, which can be as low as $25 \mathrm{~g} \mathrm{~N} /$ tree (Neilsen et al., 2009). P was fertigated for $1 \mathrm{~d}$ immediately after full bloom and before the start of regular $\mathrm{N}$ applications as ammonium polyphosphate $(10 \mathrm{~N}-15 \mathrm{P}-0 \mathrm{~K})$, which supplied $20 \mathrm{~g}$ P/tree and $13.3 \mathrm{~g} \mathrm{~N} /$ tree. Potassium was fertigated daily for 4 weeks starting 3 weeks after full bloom as potassium chloride $(0 \mathrm{~N}-0 \mathrm{P}-50 \mathrm{~K})$ to supply $20 \mathrm{~g} \mathrm{~K} /$ tree. Boron was fertigated as solubor $(20 \%$ B) daily for 4 weeks starting 1 week after full bloom to supply $0.16 \mathrm{~g} \mathrm{~B} /$ tree. Trees otherwise received standard commercial production practices [British Columbia Ministry of Agriculture and Lands (BCMAL), 2007]. These fertigation applications were continued, as described, after initiation of differential irrigation treatments.

During the 2007-09 growing seasons, a split plot experiment in a randomized complete block design was established with four irrigation treatments assigned to main plot units and three crop load treatments assigned to subplot units. There were six replicates of five tree plots (three measurement and two guard trees). The irrigation treatments included I1: $100 \%$ ET replaced daily via $2 \times$ $4-\mathrm{L} \cdot \mathrm{h}^{-1}$ emitters per tree. The irrigation quantities were a continuation of the previous atmometer-scheduled irrigation, which had been applied at $100 \%$ ET. I2: $50 \%$ ET replacement applied as in I1; I3: 50\% ET replacement applied as in $\mathrm{I} 2$ but to one side only (single emitter) of the tree; and I4: $50 \%$ ET replacement applied as in I2 except once every $2 \mathrm{~d}$. Except for I4, irrigation treatments were maintained over the 3 -year period. I4 treatments were reduced to $25 \%$ ET replacement in 2008 and $18 \%$ in 2009 both applied every second day to represent an increasingly stressful treatment. Irrigation treatments were applied throughout the growing season from May to October each year. Water applications during these months varied by year so that at $100 \%$ ET values (I1), annual per tree water applications were 1488, 1149, and 1305 L during 2007-09, respectively. Crop load treatments commenced in 2007 and were assigned to the subplot units at three levels (low, medium, and high) each year until 2009. From 2007 to 2009 , respectively, low $\left(2.5,3\right.$, and 3.75 fruits $/ \mathrm{cm}^{2}$ TCSA), medium $\left(4.5,6\right.$, and 7.5 fruits $/ \mathrm{cm}^{2}$ TCSA), and high crop loads $\left(9,12\right.$, and 15 fruits $/ \mathrm{cm}^{2}$ TCSA) crop loads were imposed from Julian day of year 160 to $169, \approx 4$ to 5 weeks after full bloom. Crop loads within categories increased over time as trees grew. Crop load adjustment was made on the same trees each year using the most recent annual TCSA values with high and low crop loads alternating from year to year.

Composite samples of 30 leaves were collected from the mid-third portion of the extension shoots of the current year's growth for each plot in mid-July each year. All samples were ovendried at $65^{\circ} \mathrm{C}$ and ground in a stainless steel mill. A sample of 25 fruit was randomly collected each year at commercial harvest for each plot. Samples were rinsed under running, distilled water and then air dried. Chemical analysis was conducted on a composite of opposite unpeeled quarters from each apple minus stem tissue and seeds. Tissue was subsequently freeze dried and ground.

For both leaf and fruit samples, $\mathrm{N}$ was determined by combustion on a $0.12-0.13 \mathrm{~g}$ sample using a Leco-FP-528 N-analyzer (LECO Corporation, St. Joseph, MI). Ca, $\mathrm{Mg}, \mathrm{B}, \mathrm{P}$, and $\mathrm{K}$ were determined by inductively coupled argon plasma spectrophotometry (Spectro Analytical Instruments, Kleve, Germany) on 0.5 g-subsamples dry ashed (leaves) or freeze dried (fruit) and dissolved in $1.2 \mathrm{~m} \mathrm{HCl}$. Leaf nutrient concentrations were expressed on a dry weight basis, whereas fruit nutrient concentrations were expressed on a fresh weight basis. Fruit nutrient removal rates (kilograms/hectare) were calculated as the product of fresh weight concentrations, tree yield, and planting density (trees/hectare).

Analysis of variance was performed on all leaf and nutrient concentrations and fruit nutrient removal rates as a split-plot design with four irrigation main plot treatments and three crop load subplots (SAS Institute Inc., 1989). Fixed effects included irrigation and crop load treatments and random effects replicates and years. Data were analyzed separately by year due to changes in crop load treatments over time, as trees grew larger in addition to the practical necessity to alternate high and low crop loads annually.

\section{Results and Discussion}

Considerable additional information concerning the response of soil moisture, plant water relations, tree growth, and fruit quality including size to imposed treatments has been reported elsewhere (Neilsen et al., 2010). This paper focuses on consequences to tree and fruit nutrition.

Leaf nutrients. In general, the range of annual overall means for leaf $\mathrm{N}, \mathrm{Ca}, \mathrm{Mg}, \mathrm{K}$, and $\mathrm{B}$ concentration indicated that average conditions throughout the 3-year experimental period were optimum with the exception of $\mathrm{N}$ and $\mathrm{K}$ (Table1). Leaf $\mathrm{N}$ concentrations, averaged overall treatments, were remarkably uniform and ranged from 27.2 to 27.4 $\mathrm{g} \cdot \mathrm{kg}^{-1}$ dry weight (dw), but were consistently above the recommended optimum range for apples (BCMAL, 2007). In contrast, annual means for leaf $\mathrm{K}$ sometimes were below $13 \mathrm{~g}$ $\mathrm{K} / \mathrm{kg} \mathrm{dw}$, the recommended lower threshold of the optimum range.

Leaf nutrient concentrations were affected by irrigation and crop load treatments but not by their interaction (Tables 2 and 3 ). Overall, the effects of crop load were more important than applied irrigation treatments: on 11 of 18 occasions ( 6 nutrients $\times 3$ years), there were significant differences among crop load treatments but differences among irrigation treatments occurred on only 4 of 18 occasions. Nitrogen, $\mathrm{Ca}$, and $\mathrm{Mg}$ are usually of sufficiently high concentrations in soil solutions that their supply to plant roots can be achieved by mass flow of water (Barber, 1984). In general, these nutrients were unaffected by irrigation treatments (Table 2). In 2009, however, leaf $\mathrm{Ca}$ concentration was elevated for all irrigation treatments relative to the minimally stressed, daily, drip irrigation treatment at $100 \%$ ET (I1). The lowest crop load reduced leaf $\mathrm{N}$ and $\mathrm{Ca}$ concentrations in 2 of 3 years. However, even at the lowest crop load, leaf $\mathrm{N}$ concentrations remained above $26.5 \mathrm{~g} \cdot \mathrm{kg}^{-1} \mathrm{dw}$, exceeding the recommended optimum range. Leaf $\mathrm{Mg}$ concentrations were unaffected by crop load and were always within the recommended range. $\mathrm{P}$ and $\mathrm{K}$ are generally considered to be supplied to plant roots via diffusion because of their low concentrations in soil solutions

Table 1. Range of average annual leaf nutrient concentration means for 'Ambrosia' apple compared with optimum range for leaf $\mathrm{N}, \mathrm{Ca}, \mathrm{Mg}, \mathrm{P}, \mathrm{K}$, and $\mathrm{B}$ in the experimental block during crop load and irrigation treatment imposition, 2007-09.

\begin{tabular}{llcc}
\hline Nutrient & \multicolumn{1}{c}{ Unit } & Range of annual means & Recommended optimum values $^{z}$ \\
\hline $\mathrm{N}$ & $\mathrm{g} \cdot \mathrm{kg}^{-1} \mathrm{dw}$ & $26.5-27.4$ & $19-24$ \\
$\mathrm{Ca}$ & $\mathrm{g} \cdot \mathrm{kg}^{-1} \mathrm{dw}$ & $15.6-17.5$ & $>13$ \\
$\mathrm{Mg}$ & $\mathrm{g} \cdot \mathrm{kg}^{-1} \mathrm{dw}$ & $3.2-3.7$ & $2.7-3.6$ \\
$\mathrm{P}$ & $\mathrm{g} \cdot \mathrm{kg}^{-1} \mathrm{dw}$ & 2.0 & $>1.5$ \\
$\mathrm{~K}$ & $\mathrm{~g} \cdot \mathrm{kg}^{-1} \mathrm{dw}$ & $11.7-15.6$ & $13-16$ \\
$\mathrm{~B}$ & $\mathrm{mg} \cdot \mathrm{kg}^{-1} \mathrm{dw}$ & $32-35$ & $31-40$ \\
\hline
\end{tabular}

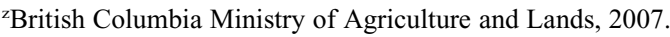

$\mathrm{dw}=$ dry weight; $\mathrm{N}=$ Nitrogen; $\mathrm{Ca}=$ Calcium; $\mathrm{Mg}=$ Magnesium; $\mathrm{P}=$ Phosphorus; $\mathrm{K}=$ Potassium; $\mathrm{B}=$ Boron. 
(Barber, 1984). Their uptake could be reduced under conditions of low soil moisture. Reduced application of water lowered leaf $\mathrm{P}$ but not leaf K concentrations (Table 3 ). Both nutrients were consistently affected by crop load with minimum concentrations always observed for trees with high crop load although leaf $\mathrm{P}$ concentrations remained within the recommended optimum range. By the third year of the study, leaf $\mathrm{K}$ concentrations for all treatments were below the optimum range $(<1.3 \mathrm{~g} \mathrm{~K} / \mathrm{kg} \mathrm{dw})$ for all but the low crop load treatments. Leaf $B$ was less affected by experimental treatments, but was reduced at $100 \%$ irrigation in 2007 and by high crop load in 2008 (Table 3). Leaf B concentrations fell below the adequacy threshold of $31 \mathrm{mg} \cdot \mathrm{kg}^{-1} \mathrm{dw}$ only in 2008 and inadequate values were therefore not consistently associated with treatments. It is also noteworthy that there were no differences in leaf nutrient concentration whether fertigated nutrients were applied to both sides (I2) as opposed to one side (I3) of the tree.

In previous studies, crop load strongly affected apple leaf concentrations for trees grown in pots with fertigation and when comparisons are made between fruiting and de-fruited trees (Hansen, 1980). Decreased leaf $\mathrm{K}$ concentration was particularly pronounced as a result of cropping of apple trees grown in pots (Jadczuk and Lenz, 1998). Decreased leaf $\mathrm{P}$ and $\mathrm{K}$ concentration and increased leaf $\mathrm{Ca}, \mathrm{Mg}$, and $\mathrm{N}$ concentration for fruiting trees have also been reported by Hansen (1973) for 'Golden Delicious'/'M.9' apple with the $\mathrm{N}$ response occurring under conditions of high $\mathrm{N}$ supply in pot studies. With the exception of leaf $\mathrm{Mg}$, these were the same patterns observed for our 'Ambrosia'/ 'M.9' trees grown under field conditions with high fertigated $\mathrm{N}$ supply. These patterns were observed despite a range of crop loads imposed in the field, none of which included complete de-fruiting. Compensatory uptake of $\mathrm{Mg}$ in leaves under low $\mathrm{K}$ supply has been observed in local fertigated orchards (Neilsen et al., 2004) and may have complicated the relationship between crop load and leaf $\mathrm{Mg}$ observed by Hansen (1973).

Deficit irrigation strategies had minimal impacts on leaf nutrient concentrations. Both leaf $\mathrm{P}$ and $\mathrm{K}$, which are transported to plant roots primarily by diffusion, were expected to be negatively impacted by less than adequate irrigation and the reduced diffusion typical of drier soils (Neilsen and Neilsen, 2003). Decreases were observed for leaf $P$ in 2 of 3 years. Fertigation, which increases soil solution nutrient concentrations, may have compensated for decreased $\mathrm{K}$ diffusion since soluble $\mathrm{K}$ was applied over a 4 -week period, 3-6 weeks postbloom. Fertigation may not have prevented the decline in leaf $\mathrm{P}$ concentration since $\mathrm{P}$ was applied on a single day immediately after full bloom. Occasionally, increased leaf $\mathrm{Ca}$ (1 year) and leaf B (1 year) were associated with deficit irrigation and may have reflected increased concentration associated with reduced vegetative growth. Imposition of irrigation deficits by restricting application of water and fertigated nutrients ( N, P, K, and B in this study) to one rather than both sides of the tree did not change leaf nutrient concentrations. This implies sufficient nutrients can be obtained from half the rooting volume, providing some flexibility in method of applying deficit irrigation in fertigated orchards.

Reduction of crop load is a useful shortterm strategy for maintaining fruit size during water shortages (Neilsen et al., 2010).

Neither the deficit irrigation treatments imposed nor reductions in crop load resulted in leaf concentrations, which signaled serious nutritional problems. Rather, the most serious issue was a decline in leaf $\mathrm{K}$ by the last year of the study, which occurred across all irrigation and crop load treatments. Although most serious for trees carrying a high crop load, these data suggested that the $20 \mathrm{~g} /$ tree $\mathrm{K}$ fertigation rate should have been increased in the latter years of the study as the trees aged.

Table 2. Leaf $\mathrm{N}, \mathrm{Ca}$, and $\mathrm{Mg}$ concentration of 'Ambrosia' apple as affected by irrigation and crop load treatment, 2007-09.

\begin{tabular}{|c|c|c|c|c|c|c|c|c|c|}
\hline \multirow[b]{2}{*}{ Irrigation treatment } & \multicolumn{3}{|c|}{ Leaf $N\left(g \cdot \mathrm{kg}^{-1} \mathrm{dw}\right)$} & \multicolumn{3}{|c|}{ Leaf $\mathrm{Ca}\left(\mathrm{g} \cdot \mathrm{kg}^{-1} \mathrm{dw}\right)$} & \multicolumn{3}{|c|}{ Leaf $\mathrm{Mg}\left(\mathrm{g} \cdot \mathrm{kg}^{-1} \mathrm{dw}\right)$} \\
\hline & 2007 & 2008 & 2009 & 2007 & 2008 & 2009 & 2007 & 2008 & 2009 \\
\hline I1 & 26.8 & 27.3 & 27.4 & 14.1 & 17.1 & $14.0 \mathrm{c}$ & 3.1 & 3.6 & 3.2 \\
\hline I2 & 27.5 & 27.2 & 27.2 & 15.4 & 19.5 & $17.0 \mathrm{ab}$ & 3.4 & 4.0 & 3.4 \\
\hline I3 & 26.8 & 26.6 & 27.2 & 15.1 & 18.5 & $15.7 \mathrm{~b}$ & 3.2 & 3.8 & 3.4 \\
\hline $\mathrm{I} 4^{\mathrm{z}}$ & 27.6 & 27.4 & 27.7 & 15.6 & 18.1 & $18.2 \mathrm{a}$ & 3.2 & 3.3 & 3.5 \\
\hline Significance & NS & NS & NS & NS & NS & $* * * *$ & NS & NS & NS \\
\hline \multicolumn{10}{|l|}{ Crop load ${ }^{y}$} \\
\hline Low & 26.7 & $26.5 \mathrm{~b}$ & $26.8 \mathrm{~b}$ & $14.5 \mathrm{~b}$ & 18.3 & $15.7 \mathrm{~b}$ & 3.2 & 3.6 & 3.2 \\
\hline Medium & 27.2 & $27.3 \mathrm{a}$ & $27.4 \mathrm{~b}$ & $15.0 \mathrm{ab}$ & 19.0 & $16.9 \mathrm{a}$ & 3.2 & 3.8 & 3.5 \\
\hline High & 27.6 & $27.7 \mathrm{a}$ & $28.0 \mathrm{a}$ & $15.6 \mathrm{a}$ & 17.5 & $16.1 \mathrm{ab}$ & 3.3 & 3.6 & 3.5 \\
\hline Significance & NS & $* * *$ & $* * *$ & $*$ & NS & $*$ & NS & NS & NS \\
\hline
\end{tabular}

${ }^{2}$ Irrigation treatment reduced to $25 \%$ evapotranspiration (ET) (2008) and 18\% ET (2009) for I4.

${ }^{y}$ Low crop load at $2.5,3$, and 3.75 fruits per $\mathrm{cm}^{2}$ trunk cross-sectional area (TCSA), 2007-09, respectively; medium crop load at 4.5, 6, and 7.5 fruits $/ \mathrm{cm}^{2}$ TCSA, 2007-09; and high crop load at 9, 12, and 15 fruits/ $\mathrm{cm}^{2}$ TCSA, 2007-09.

$*, * * *, * * * *$ Means significantly different at $P=0.05,0.001,0.0001$, respectively, or nonsignificantly (Ns) different.

$\mathrm{N}=$ Nitrogen $; \mathrm{Ca}=$ Calcium; $\mathrm{Mg}=$ Magnesium .

Table 3. Leaf $\mathrm{P}, \mathrm{K}$, and $\mathrm{B}$ concentration of 'Ambrosia' apple as affected by irrigation and crop load treatment, 2007-09.

\begin{tabular}{|c|c|c|c|c|c|c|c|c|c|}
\hline \multirow[b]{2}{*}{ Irrigation treatment } & \multicolumn{3}{|c|}{ Leaf P $\left(\mathrm{g} \cdot \mathrm{kg}^{-1} \mathrm{dw}\right)$} & \multicolumn{3}{|c|}{ Leaf K $\left(\mathrm{g} \cdot \mathrm{kg}^{-1} \mathrm{dw}\right)$} & \multicolumn{3}{|c|}{ Leaf B (mg. $\left.\mathrm{kg}^{-1} \mathrm{dw}\right)$} \\
\hline & 2007 & 2008 & 2009 & 2007 & 2008 & 2009 & 2007 & 2008 & 2009 \\
\hline I1 & $2.1 \mathrm{a}$ & $2.1 \mathrm{a}$ & 1.9 & 16.1 & 14.3 & 11.6 & $31.3 \mathrm{~b}$ & 30.1 & 32.3 \\
\hline $\mathrm{I} 2$ & $1.9 \mathrm{bc}$ & $1.9 \mathrm{bc}$ & 2.0 & 15.5 & 13.4 & 12.0 & $33.5 \mathrm{ab}$ & 29.6 & 35.2 \\
\hline $\mathrm{I} 3$ & $2.0 \mathrm{~b}$ & $2.0 \mathrm{ab}$ & 1.9 & 15.6 & 14.8 & 12.0 & $36.8 \mathrm{a}$ & 33.8 & 34.8 \\
\hline $\mathrm{I} 4^{\mathrm{z}}$ & $1.8 \mathrm{c}$ & $1.9 \mathrm{c}$ & 2.0 & 15.0 & 14.0 & 11.6 & $38.5 \mathrm{a}$ & 32.7 & 34.2 \\
\hline Significance & $* * *$ & $* *$ & NS & NS & NS & NS & $*$ & NS & NS \\
\hline \multicolumn{10}{|l|}{ Crop load ${ }^{\mathrm{y}}$} \\
\hline Low & $2.0 \mathrm{a}$ & $2.0 \mathrm{ab}$ & $2.1 \mathrm{a}$ & $16.7 \mathrm{a}$ & $14.6 \mathrm{a}$ & $13.5 \mathrm{a}$ & 35.1 & $33.5 \mathrm{a}$ & 35.3 \\
\hline Medium & $2.0 \mathrm{a}$ & $2.1 \mathrm{a}$ & $2.0 \mathrm{a}$ & $15.9 \mathrm{a}$ & $14.3 \mathrm{ab}$ & $11.7 \mathrm{~b}$ & 35.1 & $31.0 \mathrm{~b}$ & 33.6 \\
\hline High & $1.9 \mathrm{~b}$ & $1.9 \mathrm{c}$ & $1.8 \mathrm{~b}$ & $14.2 \mathrm{~b}$ & $13.5 \mathrm{~b}$ & $10.0 \mathrm{c}$ & 34.8 & $30.1 \mathrm{~b}$ & 33.4 \\
\hline Significance & ** & * & $* * *$ & $* * *$ & $*$ & $* * *$ & NS & $* *$ & NS \\
\hline
\end{tabular}

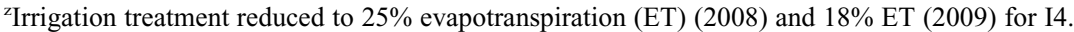

${ }^{y}$ Low crop load at $2.5,3$, and 3.75 fruits $/ \mathrm{cm}^{2}$ trunk cross-sectional area (TCSA), 2007-09, respectively; medium crop load at 4.5, 6, and 7.5 fruits $/ \mathrm{cm}^{2}$ TCSA, 2007-09; and high crop load at 9, 12, and 15 fruits/ $\mathrm{cm}^{2}$ TCSA, 2007-09.

$*, * *, * * *$ Means significantly different at $P=0.05,0.01,0.001$, respectively, or nonsignificantly (NS) different.

$\mathrm{P}=$ Phosphorus; $\mathrm{K}=$ Potassium; $\mathrm{B}=$ Boron.

Table 4. Range of average annual fruit nutrient concentration means at harvest for 'Ambrosia' apple compared with adequacy threshold (where known) for fruit $\mathrm{N}, \mathrm{Ca}, \mathrm{Mg}, \mathrm{P}, \mathrm{K}$, and $\mathrm{B}$ during crop load and treatment imposition, 2007-09.

\begin{tabular}{llcc}
\hline Nutrient & \multicolumn{1}{c}{ Unit } & Range of annual means & Adequacy thresholdz $^{\mathrm{z}}$ \\
\hline $\mathrm{N}$ & $\mathrm{g} \cdot \mathrm{kg}^{-1}$ fw & $0.56-0.58$ & $\mathrm{nd}^{\mathrm{y}}$ \\
$\mathrm{Ca}$ & $\mathrm{g} \cdot \mathrm{kg}^{-1}$ fw & $0.038-0.058$ & $>0.040$ \\
$\mathrm{Mg}$ & $\mathrm{g} \cdot \mathrm{kg}^{-1} \mathrm{fw}$ & $0.057-0.058$ & $\mathrm{nd}$ \\
$\mathrm{P}$ & $\mathrm{g} \cdot \mathrm{kg}^{-1} \mathrm{fw}$ & $0.103-0.111$ & $>0.10$ \\
$\mathrm{~K}$ & $\mathrm{~g} \cdot \mathrm{kg}^{-1} \mathrm{fw}$ & $1.14-1.24$ & $>1.0$ \\
$\mathrm{~B}$ & $\mathrm{mg} \cdot \mathrm{kg}^{-1}$ fw & $3.0-3.1$ & nd \\
\hline
\end{tabular}

${ }^{\mathrm{z}}$ British Columbia Ministry of Agriculture and Lands, 2007.

${ }^{y}$ Not determined.

$\mathrm{N}=$ Nitrogen $; \mathrm{Ca}=$ Calcium; $\mathrm{Mg}=$ Magnesium $; \mathrm{P}=$ Phosphorus $; \mathrm{K}=$ Potassium $; \mathrm{B}=\mathrm{Boron} ; \mathrm{fw}=\mathrm{fresh}$ weight. 
Table 5. Fruit N, Ca, and Mg concentration at harvest of 'Ambrosia' apple as affected by irrigation and crop load treatment, $2007-09$.

\begin{tabular}{|c|c|c|c|c|c|c|c|c|c|c|c|c|c|c|c|c|c|}
\hline \multirow[b]{3}{*}{ Irrigation treatment } & \multicolumn{5}{|c|}{ Fruit $\mathrm{N}\left(\mathrm{g} \cdot \mathrm{kg}^{-1} \mathrm{fw}\right)$} & \multicolumn{5}{|c|}{ Fruit Ca $\left(\mathrm{g} \cdot \mathrm{kg}^{-1} \mathrm{fw}\right)$} & \multicolumn{7}{|c|}{ Fruit $\mathrm{Mg}\left(\mathrm{g} \cdot \mathrm{kg}^{-1} \mathrm{fw}\right)$} \\
\hline & \multirow[b]{2}{*}{2007} & \multicolumn{3}{|c|}{2008} & \multirow[b]{2}{*}{2009} & \multirow[b]{2}{*}{2007} & \multicolumn{3}{|c|}{2008} & \multirow[b]{2}{*}{2009} & \multirow[b]{2}{*}{2007} & \multicolumn{3}{|c|}{2008} & \multicolumn{3}{|c|}{2009} \\
\hline & & CL1 & CL2 & CL3 & & & CL1 & CL2 & CL3 & & & CL1 & CL2 & CL3 & CL1 & CL2 & CL3 \\
\hline I1 & 0.56 & 0.53 & 0.44 & 0.47 & 0.54 & 0.035 & 0.034 & 0.038 & 0.040 & $0.045 \mathrm{c}$ & $0.056 \mathrm{c}$ & 0.057 & 0.050 & 0.049 & 0.057 & 0.052 & 0.047 \\
\hline $\mathrm{I} 2$ & 0.60 & 0.54 & 0.54 & 0.59 & 0.58 & 0.039 & 0.036 & 0.046 & 0.052 & $0.056 \mathrm{~b}$ & $0.060 \mathrm{a}$ & 0.059 & 0.056 & 0.056 & 0.055 & 0.058 & 0.056 \\
\hline $\mathrm{I} 3$ & 0.57 & 0.48 & 0.51 & 0.54 & 0.54 & 0.038 & 0.034 & 0.040 & 0.046 & $0.054 \mathrm{~b}$ & $0.058 \mathrm{bc}$ & 0.056 & 0.057 & 0.053 & 0.059 & 0.057 & 0.054 \\
\hline $\mathrm{I} 4^{\mathrm{z}}$ & 0.60 & 0.62 & 0.69 & 0.75 & 0.64 & 0.040 & 0.043 & 0.061 & $0.070 \mathrm{a}$ & $0.077 \mathrm{a}$ & $0.059 \mathrm{ab}$ & 0.061 & 0.065 & 0.064 & 0.063 & 0.063 & 0.067 \\
\hline Significance & NS & & & & NS & NS & & & & $* * * *$ & $*$ & & & & & & \\
\hline \multicolumn{18}{|l|}{$\mathrm{CL}^{\mathrm{y}}$} \\
\hline Low & $0.64 \mathrm{a}$ & & & & 0.56 & $0.032 \mathrm{c}$ & & & & $0.043 \mathrm{c}$ & $0.062 \mathrm{a}$ & & & & & & \\
\hline Medium & $0.57 \mathrm{~b}$ & & & & 0.57 & $0.037 \mathrm{bc}$ & & & & $0.058 \mathrm{~b}$ & $0.058 \mathrm{~b}$ & & & & & & \\
\hline High & $0.53 \mathrm{c}$ & & & & 0.59 & $0.046 \mathrm{a}$ & & & & $0.073 \mathrm{a}$ & $0.054 \mathrm{c}$ & & & & & & \\
\hline Significance & $* * * *$ & & & & NS & $* * * *$ & & & & $* * * *$ & $* * * *$ & & & & & & \\
\hline $\mathrm{CL} \times \mathrm{I}$ interaction & NS & & $*$ & & NS & NS & & $*$ & & & & & $*$ & & & $* *$ & \\
\hline$\underline{\text { SE interaction }}$ & & & 0.03 & & & & & 0.05 & & & & & 0.002 & & & 0.002 & \\
\hline
\end{tabular}

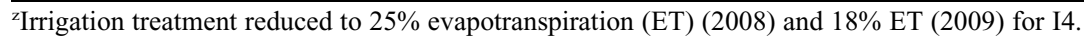

${ }^{\mathrm{y}}$ Low crop load at $2.5,3$, and 3.75 fruits $/ \mathrm{cm}^{2}$ trunk cross-sectional area (TCSA), 2007-09, respectively; medium crop load at $4.5,6$, and 7.5 fruits/ $\mathrm{cm}^{2}$ TCSA, 2007-09; and high crop load at 9, 12, and 15 fruits $/ \mathrm{cm}^{2}$ TCSA, 2007-09.

*,**,***Means significantly different at $P=0.05,0.01,0.0001$, respectively, or nonsignificantly (NS) different.

$\mathrm{CL}=$ crop load; $\mathrm{fw}=$ fresh weight; $\mathrm{N}=$ Nitrogen; $\mathrm{Ca}=$ Calcium; $\mathrm{Mg}=$ Magnesium; $\mathrm{I}=$ Irrigation treatment.

Table 6. Fruit P, K, and B concentration at harvest of 'Ambrosia' apple as affected by irrigation and crop load treatment, $2007-09$.

\begin{tabular}{|c|c|c|c|c|c|c|c|c|c|c|c|}
\hline \multirow{2}{*}{ Irrigation treatment } & \multirow{2}{*}{\multicolumn{3}{|c|}{ Fruit $P\left(g \cdot \mathrm{kg}^{-1} \mathrm{fw}\right)$}} & \multirow{2}{*}{\multicolumn{3}{|c|}{ Fruit $\mathrm{K}\left(\mathrm{g} \cdot \mathrm{kg}^{-1} \mathrm{fw}\right)$}} & \multicolumn{5}{|c|}{ Fruit B $\left(g \cdot \mathrm{kg}^{-1} \mathrm{fw}\right)$} \\
\hline & & & & & & & 2007 & 2008 & \multicolumn{3}{|c|}{2009} \\
\hline I1 & 0.12 & $0.10 \mathrm{~b}$ & 0.10 & 12.4 & $10.4 \mathrm{c}$ & $10.3 \mathrm{c}$ & 2.5 & $2.6 \mathrm{c}$ & 3.8 & 2.4 & 2.3 \\
\hline I3 & 0.11 & $0.10 \mathrm{~b}$ & 0.10 & 12.6 & $11.7 \mathrm{~b}$ & $11.2 \mathrm{c}$ & 3.2 & $3.2 \mathrm{ab}$ & 3.5 & 3.1 & 3.0 \\
\hline $\mathrm{I} 4^{\mathrm{z}}$ & 0.10 & $0.11 \mathrm{a}$ & 0.11 & 12.4 & $12.9 \mathrm{a}$ & $12.9 \mathrm{a}$ & 3.7 & $3.4 \mathrm{a}$ & 3.4 & 3.3 & 3.5 \\
\hline Significance & NS & $*$ & NS & NS & $* * * *$ & $* * * *$ & NS $(0.06)$ & $*$ & & & \\
\hline Medium & $0.11 \mathrm{~b}$ & $0.10 \mathrm{~b}$ & $0.10 \mathrm{~b}$ & $12.5 \mathrm{~b}$ & $11.5 \mathrm{~b}$ & $11.3 \mathrm{~b}$ & $3.1 \mathrm{ab}$ & $2.8 \mathrm{~b}$ & & & \\
\hline High & $0.09 \mathrm{c}$ & $0.10 \mathrm{~b}$ & $0.09 \mathrm{c}$ & $10.5 \mathrm{c}$ & $10.6 \mathrm{c}$ & $9.9 \mathrm{c}$ & $2.8 \mathrm{~b}$ & $2.5 \mathrm{c}$ & & & \\
\hline Significance & $* * * *$ & $* * *$ & $* * * *$ & $* * * *$ & $* * * *$ & $* * * *$ & $*$ & $* * * *$ & & & \\
\hline $\begin{array}{l}\mathrm{CL} \times \mathrm{I} \text { interaction } \\
\mathrm{SE} \text { interaction }\end{array}$ & NS & NS & NS & NS & NS & NS & NS & NS & & $\begin{array}{c}* \\
0.2\end{array}$ & \\
\hline
\end{tabular}

Irrigation treatment reduced to 25\% evapotranspiration (ET) (2008) and 18\% ET (2009) for I4.

${ }^{{ }^{y} L o w}$ crop load at $2.5,3$, and 3.75 fruits $/ \mathrm{cm}^{2}$ trunk cross-sectional area (TCSA), 2007-2009, respectively; medium crop load at $4.5,6$, and 7.5 fruits/ $\mathrm{cm}^{2}$ TCSA, 2007-09; and high crop load at 9, 12, and 15 fruits $/ \mathrm{cm}^{2}$ TCSA, 2007-09.

$*, * * *, * * * *$ Means significantly different at $P=0.05, P=0.001$, or $P=0.0001$, respectively, or nonsignificantly (Ns) different.

$\mathrm{CL}=$ crop load; $\mathrm{fw}=$ fresh weight; $\mathrm{P}=$ Phosphorus; $\mathrm{K}=$ Potassium; $\mathrm{B}=$ Boron; $\mathrm{I}=$ Irrigation treatment.

Fruit nutrients. The relative range of annual means of all treatments was least for $\mathrm{N}, \mathrm{Mg}$, and $\mathrm{B}$ and widest for Ca (Table 4). Adequacy thresholds are less developed for fruit compared with leaf nutrient concentrations. For those nutrients for which standards have been developed, only fruit $\mathrm{Ca}$ appeared to be below optimum in some study years.

Fruit nutrient concentrations were affected by irrigation ( 6 of 18 occasions), crop load (12 of 18 occasions), or their interaction (5 of 18 ocassions) (Tables 5 and 6 ) indicating a complex interaction among treatments. Fruit $\mathrm{N}$ was minimally affected by irrigation treatment (Table 5). Irrigation main effects were only measured in 1 year for fruit $\mathrm{Ca}$ (2009) and Mg (2007) (Table 5). In both these instances, the lowest concentrations were observed for $100 \%$ ET irrigation (I1) and the highest concentrations were observed for I4. Fruit $\mathrm{N}$ was inconsistently affected by crop load being the highest for low crop load trees in 2007, unaffected by crop load in 2009 and highest for highly cropped trees but only at I4 in 2008 (significant crop load $\times$ irriga- tion interaction, Table 5). Fruit $\mathrm{Ca}$ was consistently lowest for low-cropped trees, even in 2008 when there was significant interaction between irrigation and crop load. In this year, trees with low crop loads had significantly lower fruit $\mathrm{Ca}$ concentrations than trees with high crop load, except for irrigation treatment I1. Fruit Ca concentrations below the $0.040 \mathrm{~g} \mathrm{Ca} / \mathrm{kg}$ fresh weight threshold were frequent among treatments in 2007, the first year of the study. Suboptimal fruit $\mathrm{Ca}$ concentrations were also observed in 2008 for lightly cropped trees under all irrigation treatments and in mediumcropped trees at I1. Fruit Ca concentrations were uniformly high in 2009. Fruit $\mathrm{Mg}$ decreased with increased crop load in 2007 but this effect was only consistently observed at I1 in 2008 and 2009 when crop load $x$ irrigation interactions occurred. There were minor and inconsistent differences for fertigated nutrients in fruit nutrient concentration between I2 and I3 (applied one side) treatments with $\mathrm{I} 3$ values lower for fruit $\mathrm{K}$ in 1 year and either lower or higher for fruit $\mathrm{B}$ (depending on crop load) in 1 year.
For the nutrients $\mathrm{P}, \mathrm{K}$, and $\mathrm{B}$, crop load had the dominant effect on fruit concentration (Table 6). High crop load consistently decreased fruit $\mathrm{P}, \mathrm{K}$, and $\mathrm{B}$ concentrations. This pattern was observed for B across irrigation treatments except I4 in 2009, when a significant crop load $\times$ irrigation interaction occurred. When irrigation effects were significant (2008 for P, K, and B and 2009 for K) highest concentrations occurred under the most stressful irrigation treatment (I4).

In general, deficit irrigation has been reported to have limited effects on fruit mineral nutrition although changes are considered to result in improved fruit quality (Behboudian and Mills, 1997). Effects were similarly minimal in our study: the most severe of deficit irrigation treatments (I4), when significant, usually increased fruit nutrient concentration. This suggests that reductions in fruit size were greater than reductions in nutrient inflow to the fruit thereby increasing fruit nutrient concentration. Since fruit $\mathrm{Ca}$ concentration increases were proportionally larger than any increases in fruit $\mathrm{K}$, fruit $\mathrm{K} / \mathrm{Ca}$ ratios, which have been 
A)

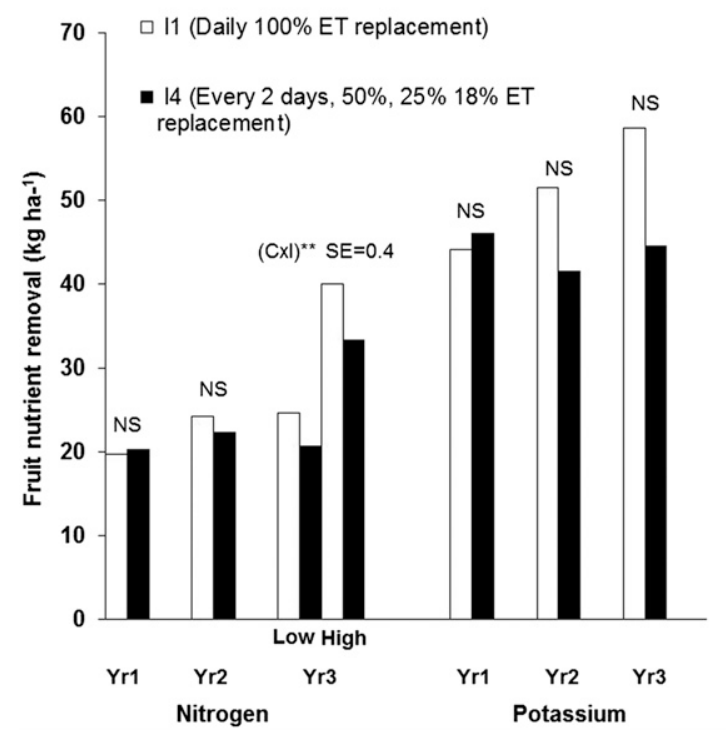

B)

$\square$ Crop Load Low

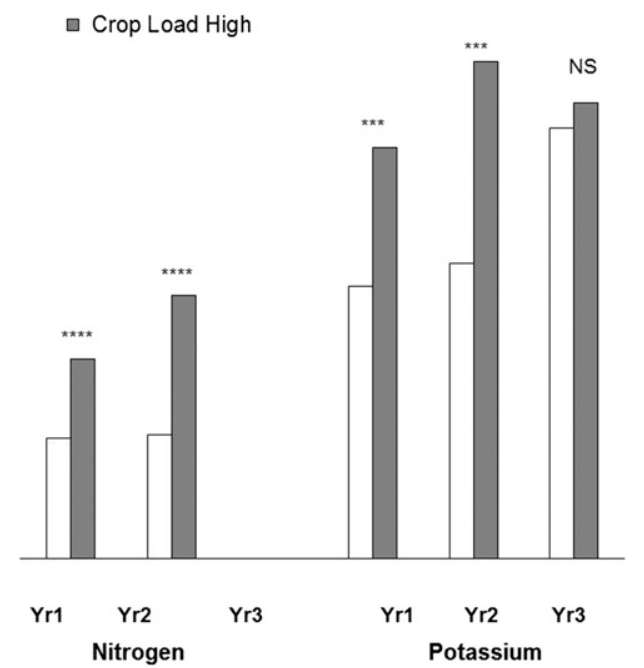

Fig. 1. Unit area $\left(\mathrm{kg} \cdot \mathrm{ha}^{-1}\right)$ fruit $\mathrm{N}$ and $\mathrm{K}$ removal rates as affected by (A) irrigation strategy (I1 vs. I4) and (B) crop load (low vs. high) during a 3-year study. Means are significantly different at $P=0.01(* *), P=0.001(* * *), P=0.0001(* * * *)$, or not significantly different (NS).

associated with decreased fruit storage potential when too high (Wolk et al., 1998), were not increased. The minor effects on fruit nutrient concentration of restricting water and fertilizer application to one side of the tree is further evidence of minimal nutritional consequences of reducing water application by reducing the volume of wetted soil.

Crop load has previously been identified as a major factor influencing fruit mineral concentration (Hansen, 1980). Volz et al. (1993) reported low-crop trees produced fruit with decreased $\mathrm{Ca}$ and increased $\mathrm{K}$ concentration with more fruit disorders. Ferguson and Watkins (1992) reported low crop load decreased fruit $\mathrm{Ca}$, increased fruit $\mathrm{K}$, and did not affect fruit $\mathrm{Mg}$. Our results also suggest that fruit nutrient concentrations, except for $\mathrm{Ca}$, were highest when crop loads were minimal. Collectively, these results indicate that for growers adopting deficit irrigations strategies and reducing crop load to maintain fruit size, there is a possibility of Ca-related disorders, particularly for susceptible cultivars in the first year of crop load adjustment if trees are young with an initial low crop load.

Fruit nutrient removal rates. Potassium and $\mathrm{N}$ have the highest measured nutrient concentrations in harvested fruit (Table 4) and the largest unit area nutrient removal rates when calculations were made to account for tree yield and spacing (Fig. 1). When the effects of treatment extremes of irrigation (I1 vs. I4) and crop load (high vs. low) were compared, crop load had a stronger influence on the amount of $\mathrm{N}$ and $\mathrm{K}$ removed with fruit (Fig. 1). Comparing the irrigation extremes, $\mathrm{N}$ removal rate was unaffected by irrigation treatment in the first 2 years. $\mathrm{N}$ removal rate was greater at I1 relative to I4 in the last year of the study (2009), at high crop load only when there was a significant crop load $x$ irrigation interaction. In contrast, $\mathrm{N}$ and $\mathrm{K}$ removal rates were greater at high relative to low crop load in the first 2 years of the study.

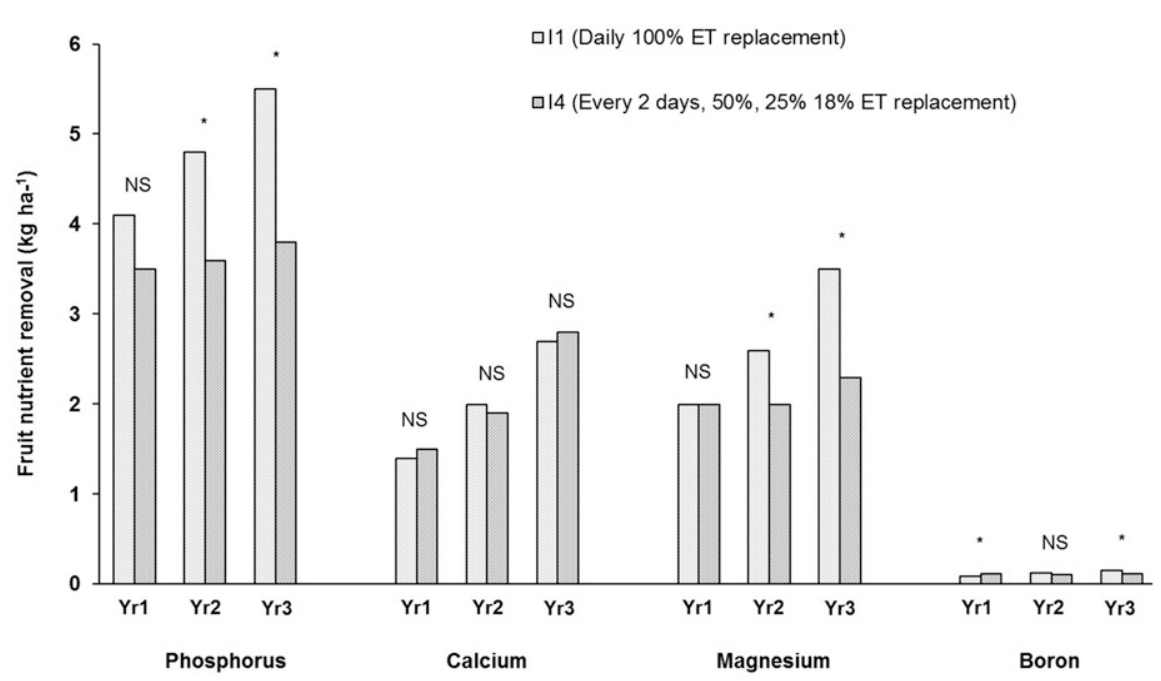

Fig. 2. Unit area $\left(\mathrm{kg} \cdot \mathrm{ha}^{-1}\right)$ fruit $\mathrm{P}, \mathrm{Ca}, \mathrm{Mg}, \mathrm{B}$ removal rates as affected by irrigation strategy (I1 vs. I4) during a 3-year study. Means are significantly different at $P=0.05\left(^{*}\right)$, or not significantly different (NS).

Irrigation effects were more apparent for the nutrients $\mathrm{P}, \mathrm{Ca}, \mathrm{Mg}$, and $\mathrm{B}$, which had lower unit area fruit nutrient removal rates (Fig. 2). For 2 years each for $\mathrm{P}, \mathrm{Mg}$, and $\mathrm{B}$ significantly higher nutrient removal rates were associated with $100 \%$ ET (I1) rather than deficit irrigation (I4). Calcium was the only nutrient where removal rate was consistently unaffected by deficit irrigation. As with $\mathrm{N}$ and $\mathrm{K}$, crop load strongly influenced nutrient removal rate, with $\mathrm{Ca}$ and $\mathrm{Mg}$ removal consistently higher at high crop load and $\mathrm{P}$ and $\mathrm{B}$ removal higher in the first 2 years of the study (Fig. 3).

The range of fruit annual nutrient removal rates across treatments relative to annual fertilizer application rates during the 3-year study indicated no nutrient removal rates exceeded the rate of applied fertilizer (Table 7). There were, however, differences in the proportion of annual fertilizer applications removed with fruit among nutrients with $\mathrm{P}$ removals the lowest and $\mathrm{K}$ removals the highest of the fertigated nutrients. Intermediate proportions of fertilizer $\mathrm{N}$ and $\mathrm{B}$ were removed. In addition to fruit nutrient removal, annual nutrient requirements for dwarf apple trees include a considerable amount of nutrients contained in shoots and leaves. A recent study by Cheng and Raba (2009) confirmed a relatively high proportion of total annual $\mathrm{K}$ demand associated with fruit $(\approx 70 \%)$ for 'Gala'/'M.26' rootstock grown in sand culture. Furthermore, their study indicated more nutrients are removed in fruit than leaves and shoots for B $(76 \%)$ and $\mathrm{P}(61 \%)$. In contrast, $\mathrm{N}$ demands are less for fruit (37.5\% of total) than for shoots and leaves, whose annual $\mathrm{N}$ requirements are also supplied by $\mathrm{N}$ remobilized from tree reserve $\mathrm{N}$. If fruit nutrient contents occur as a similar proportion to shoot plus leaf requirements in 'Ambrosia' as for 'Gala', then maximum annual total (fruit + leaf + shoot) nutrient demands observed in our study exceeded 


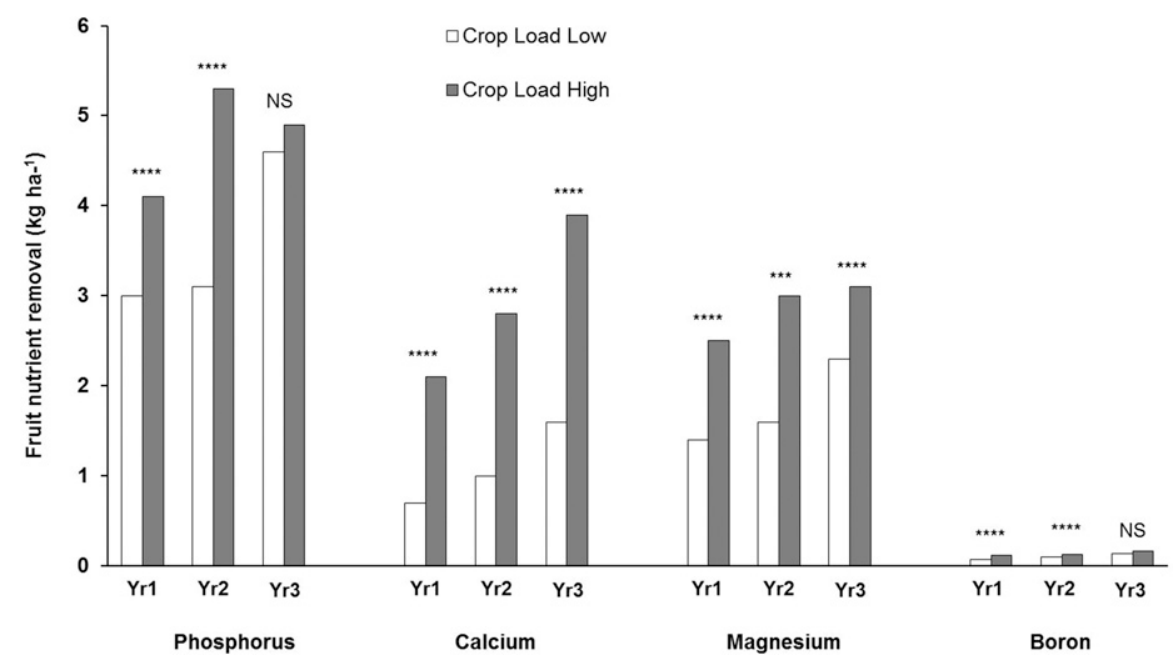

Fig. 3. Unit area $\left(\mathrm{kg} \cdot \mathrm{ha}^{-1}\right)$ fruit $\mathrm{P}, \mathrm{Ca}, \mathrm{Mg}, \mathrm{B}$ removal rates as affected by crop load (low vs. high) during a 3-year study. Means significantly different at $P=0.001(* * *), P=0.0001(* * * *)$, or not significantly different (NS)

Table 7. Annual N-P-K fertigation rates and range of annual fruit nutrient removal rates per tree and as a percent of applied.

\begin{tabular}{lccc}
\hline Nutrient & Fertigation rate $\left(\mathrm{kg} \cdot \mathrm{ha}^{-1}\right)$ & Fruit removal rate $\left(\mathrm{kg} \cdot \mathrm{ha}^{-1}\right)$ & Fruit removal $(\%$ applied $)$ \\
\hline $\mathrm{N}$ & 238 & $19-41$ & $8-17$ \\
$\mathrm{P}$ & 63.5 & $3.5-5.4$ & $5.5-8.5$ \\
$\mathrm{~K}$ & 63.5 & $32-59$ & $66-93$ \\
$\mathrm{~B}$ & 0.57 & $0.09-0.15$ & $16-26$ \\
\hline
\end{tabular}

$\mathrm{N}=$ Nitrogen; $\mathrm{P}=$ Phosphorus; $\mathrm{K}=$ Potassium; $\mathrm{B}=$ Boron.

fertilizer applications only for $\mathrm{K}(130 \%$ of annual fertigation rates) but not for $\mathrm{P}(14 \%)$, B (37\%), or N (48\%). This suggests that for the experimental treatments tested in this study, $\mathrm{K}$ fertigation rates should have been higher as the trees aged, which is consistent with the decline in leaf $\mathrm{K}$ concentrations to inadequate values by the third year across all treatments. In contrast, it might be argued that excessive $\mathrm{P}$ applications were made during the study. However, the susceptibility of $P$ to precipitation within the soil (Neilsen et al., 1993; O'Neil et al., 1979) and documented benefits of large $\mathrm{P}$ applications at bloom (Neilsen et al., 2008) support maintenance of these $\mathrm{P}$ application rates. However, reductions in the $\mathrm{B}$ and $\mathrm{N}$ application rates may have been possible. Zanotelli et al. (2014) conducted a field study with sequential destructive sampling of bourse shoots to model the seasonal dynamics of leaf and fruit nutrient uptake. Their study also found a dominant role for fruit in whole tree nutrient uptake by the time of harvest. They also measured a variation in the strength of the fruit demand within the season suggesting the possibility of using more detailed within season information to vary fertigation strategies within the year.

Adopting conservative deficit irrigation strategies should not create excessive nutritional requirements since growth of shoots and leaves is, if anything, reduced and fruit nutrient removals (especially for $\mathrm{P}, \mathrm{Mg}$, and $\mathrm{B}$ ) are reduced relative to full irrigation Also, uptake of $\mathrm{K}$ and $\mathrm{Mg}$ appears to be relatively independent of the irrigation regimes tested. A dominant effect on nutrient removal rate was crop load: higher crop loads increased nutrient uptake rates for all nutrients measured in this study (N, K, Ca, Mg, and B). Since one of the strategies of maintaining fruit size under imposed drought is to reduce crop load (Neilsen et al., 2010), this has the additional consequence of reducing nutrient demand. Thus, plantings with a successful fertigation strategy before drought imposition will not require additional fertilization when droughted.

It is noteworthy that $\mathrm{Ca}$ is usually not applied to the soil in orchards, and is critical for optimizing fruit quality (Vang-Petersen, 1980). Fruit uptake of Ca was unaffected by the various deficit irrigation strategies but was strongly affected by crop load. Adequate soil moisture content is considered critical for $\mathrm{Ca}$ accumulation in fruit (Faust, 1989) but these results would suggest that increased crop load has a more important role in the quantity of $\mathrm{Ca}$ taken up by the fruit. In part, this could reflect a minimal effect of deficit irrigation on early season soil moisture contents during the critical early season $\mathrm{Ca}$ uptake period. In spring, ET demand is often reduced by cool temperatures and soil moisture recharged by cumulative over-winter precipitation. A strategy of reducing crop load under deficit irrigation to maintain fruit size would also reduce fruit $\mathrm{Ca}$ uptake. Quality problems with low fruit Ca concentration could result for cultivars like Braeburn and Honeycrisp (Peryea et al., 2007), which are particularly susceptible to $\mathrm{Ca}$ related disorders when lightly cropped.

\section{Conclusions}

In this 3-year comparative study, variations in crop load had greater effects on apple tree nutrition than any of the deficit irrigation strategies imposed on 'Ambrosia' apple trees receiving a recommended NPKB fertigation program. Midseason leaf concentrations, which are routinely used as an indicator of tree nutritional status, were infrequently affected by deficit irrigation with the exception of leaf $P$, which was generally decreased by reductions in water application. In contrast, crop load had a greater influence on leaf concentrations. Effects, however, varied by nutrient with $\mathrm{Mg}$ and $\mathrm{B}$ minimally affected, $\mathrm{N}$ and $\mathrm{Ca}$ concentration generally increased, and $\mathrm{P}$ and $\mathrm{K}$ consistently decreased at highest crop load. Despite changes in leaf concentration observed in response to treatments, deficient values were only measured for $\mathrm{K}$ regardless of treatment by third year. This implied that the $20 \mathrm{~g} \mathrm{~K} /$ tree fertigation rate was marginal on initiation of the experiment and inadequate for optimum $\mathrm{K}$ nutrition as the trees aged.

The imposition of conservative irrigation strategies reduced fruit size more than fruit nutrient accumulation as indicated by maximum fruit $\mathrm{Ca}, \mathrm{Mg}, \mathrm{P}, \mathrm{K}$, and $\mathrm{B}$ concentrations, when significantly affected, occurring in the smallest fruit when the lowest amount of water was applied. Crop load had multiyear effects on fruit $\mathrm{Ca}, \mathrm{B}, \mathrm{P}$, and $\mathrm{K}$ concentrations with $\mathrm{B}, \mathrm{P}$, and $\mathrm{K}$ concentrations decreased at high crop load, contrasting with minimum fruit $\mathrm{Ca}$ concentration at low crop load. This implies that deficit irrigation strategies could result in inadequate fruit $\mathrm{Ca}$ concentrations if crop load is light.

Fruit nutrient removal rates, as expressed in kilograms per hectare, were often reduced by deficit irrigation strategies for $\mathrm{P}, \mathrm{Mg}$, and $\mathrm{B}$ and unaffected by irrigation for $\mathrm{Ca}$ and $\mathrm{K}$. In contrast, high crop load increased fruit nutrient removal rate for all measured nutrients in most years, indicating crop load had a dominant effect on fruit nutrient removal rates. Deficit irrigation applied with the additional strategy of reducing crop load to maintain fruit size reduces fertilizer requirements. Furthermore, with the exception of K, the original NPKB fertigation applications were in excess of tree needs.

\section{Literature Cited}

Allen, R.G., L.S. Pereira, D. Raes, and M. Smith. 1998. Crop evapotranspiration. Guidelines for computing crop water requirements. FAO Irrigation and Drainage Paper 56. FAO, Rome, Italy.

Barber, S.A. 1984. Soil nutrient bioavailability, a mechanistic approach. Wiley, New York, NY.

Barnett, T., R. Malone, W. Penell, D. Stammer, B. Semtner, and W. Washington. 2004. The effects of climate change on water resources in the west: Introduction and overview. Clim. Change 62:1-11.

Behboudian, M.H. and T.M. Mills. 1997. Deficit irrigation in deciduous orchards. Hort. Rev. 21:105-131.

British Columbia Ministry of Agriculture and Lands (BCMAL). 2007. Integrated fruit production guide for commercial fruit growers. Interior Districts, Victoria, British Columbia, Canada.

Brun, C.A., J.T. Raese, and E.A. Stahly. 1985. Seasonal response of 'Anjou' pear trees to different irrigation regimes. II Mineral composition of 
fruit and leaves, fruit disorders and fruit set. J. Amer. Soc. Hort. Sci. 110:835-840.

Cheng, L. and R. Raba. 2009. Accumulation of macroand micronutrients and nitrogen demand-supply relationship of 'Gala'/'Malling26' apple trees grown in sand culture. J. Amer. Soc. Hort. Sci. 134:3-13.

Fallahi, E., B. Fallahi, B. Shafi, D. Neilsen, and G. Neilsen. 2011. The impact of long-term evapotranspiration-based water scheduling in various irrigation regimes on tree growth, yield and fruit quality at harvest in 'Fugi' apple. J. of Amer. Pom. Soc. 65:42-53.

Faust, M. 1989. Physiology of temperate zone fruit trees. Wiley, New York, NY.

Ferguson, I.B. and C.B. Watkins. 1992. Crop load affects mineral concentrations and incidence of bitter pit in 'Cox's Orange Pippin' apple fruit. J. Amer. Soc. Hort. Sci. 117:373-376.

Hansen, P. 1973. The effect of cropping on the growth and uptake of nutrients by apple trees at different levels of Nitrogen, potassium, magnesium and phosphorus. Acta. Agr. Scandinavica 23:87-92.

Hansen, P. 1980. Crop load and nutrient translocation, p. 201-212. In: D. Atkinson, J.E. Jackson, R.O. Sharples, and W.M. Waller (eds.). Mineral nutrition of fruit trees. Buttersworth, London, UK.

Jadczuk, E. and F. Lenz. 1998. Effect of water supply and fruit load on uptake and distribution of potassium in 'Golden Delicious' trees. Gartenbauwissenschaft 63:193-196.

Mills, T.M., M.H. Behboudian, and B.E. Clothier. 1996. Water relations, growth, and the composition of 'Braeburn' apple fruit under deficit irrigation. J. Amer. Soc. Hort. Sci. 121:286-291.

Mpelasoka, B.S., M.H. Behboudian, and S. Ganesh. 2001. Fruit quality attributes and their interrelationships of 'Braeburn' apple in response to deficit irrigation and to crop load. Gartenbauwissenschaft 66:247-253.

Naor, A., I. Klein, I. Doron, Y. Gal, Z. Ben-David, and B. Bravdo. 1997. The effect of irrigation and crop load on stem water potential and apple fruit size. J. Hort. Sci. 72:765-771.

Naschitz, S. and A. Naor. 2005. The effect of crop load on tree water consumption of 'Golden Delicious' apples in relation to fruit size: An operative model. J. Amer. Soc. Hort. Sci. 130:7-11.

Neilsen, D., G.H. Neilsen, L. Herbert, and S. Guak. 2010. Effect of irrigation and crop load management on fruit nutrition and quality for Ambrosia/M.9 apple. Acta Hort. 868:63-72.

Neilsen, D., P. Parchomcuk, and E.J. Hogue. 1993. Soil and peach seedling responses to soluble phosphorus applied in single or multiple doses. Commun. Soil Sci. Plant Anal. 24:881-898.

Neilsen, D., C.A.S. Smith, G. Frank, W. Koch, Y. Alila, W.S. Merritt, W.G. Taylor, M. Barton, J. W. Hall, and S.J. Cohen. 2006. Potential impacts of climate change on water availability for crops in the Okanagan Basin, British Columbia. Can. J. Soil Sci. 86:921-936.

Neilsen, D., S. Smith, T. Van der Gulik, B. Taylor, A. Cannon, and R. Fretwell. 2015. Modeling regional water demand for current and future climate in the Okanagan Basin, British Columbia, Canada. Acta Hort. 1068:211-218.

Neilsen, G.H. and D. Neilsen. 2003. Nutritional requirements of apple, p. 267-302. In: D.C. Ferree and I.J. Warrington (eds.). Apples, production and uses. CABI Publishing, Oxon, UK.

Neilsen, G.H., D. Neilsen, and L. Herbert. 2009. Nitrogen fertigation and timing of application affect nitrogen nutrition, yield, firmness and color of apples grown at high density. HortScience 44:1425-1431.

Neilsen, G.H., D. Neilsen, L.C. Herbert, and E.J. Hogue. 2004. Response of apple to fertigation of $\mathrm{N}$ and $\mathrm{K}$ under conditions susceptible to the development of K deficiency. J. Amer. Soc. Hort. Sci. 129:26-31.

Neilsen, G.H., D. Neilsen, P. Toivonen, and L. Herbert. 2008. Annual bloom-time phosphorus fertigation affects soil phosphorus, apple tree phosphorus nutrition, yield and fruit quality. HortScience 43:885-890.

O’Neil, M.K., B.R. Gardner, and R.L. Roth. 1979. Orthophosphoric acid as a phosphorus fertilizer in trickle irrigation. Soil Sci. Soc. Amer. J. 43:283-286

Payne, J.T., A.W. Wood, A.F. Hamlet, R.N. Palmer, and D.P. Lettenmaier. 2004. Mitigating the effects of climate change on the resources of the Columbia River Basin. Clim. Change 62:233-256.
Parchomchuk, P., R.C. Berard, and T.W. Van der Gulik. 1996. Automatic irrigation scheduling using an electronic atmometer, p. 1099-1104. In: C.R. Camp, E.J. Sadler, and R.E. Yordu (eds.). Evapotranspiration and irrigation scheduling. Amer. Soc. Agr. Engn. Proc. Intl. Conf., San Antonio, TX.

Peryea, F.J., G.H. Neilsen, and D. Faubion. 2007. Start-timing for calcium chloride spray programs influences fruit calcium and bitter pit in 'Braeburn' and 'Honeycrisp' apples. J. Plant Nutr. 30:1213-1227.

Proebsting, E.J., Jr., and J.E. Middleton. 1980. The behavior of peach and pear trees under extreme drought stress. J. Amer. Soc. Hort. Sci. 105:380-385.

Proebsting, E.J., Jr., J.E. Middleton, and M.O. Mahan. 1981. Performance of bearing cherry and prune trees under very low irrigation rates. J. Amer. Soc. Hort. Sci. 106:243-246.

SAS Institute Inc. 1989. SAS/STAT user's guide. Version 6, Vol. 2. SAS Institute Inc., Cary, NC.

Vang-Petersen, O. 1980. Calcium nutrition of apple trees: A review. Sci. Hort. 12:1-9.

Volz, R.K., I.B. Ferguson, J.H. Bowen, and C.B. Watkins. 1993. Crop load effects on fruit mineral nutrition, maturity, fruiting and tree growth of 'Cox's Orange Pippin' apple. J. Hort. Sci. 68:127-137.

Wittneben, U. 1986. Soils of the Okanagan and Similkameen Valleys. Ministry of Environment Technical Report 10. British Columbia Soil Survey, Victoria, British Columbia, Canada.

Wolk, W.D., O.L. Lau, G.H. Neilsen, and B.G. Drought. 1998. Factors and time of sample collection for correlating storage potential of 'McIntosh', 'Spartan' and 'Golden Delicious' apples. J. Amer. Soc. Hort. Sci. 123:104-109.

Wünsche, J.N., J.W. Palmer, and D.H. Greer. 2000. Effects of crop load on fruiting and gasexchange characteristics of 'Braeburn'/ M.26 apple trees at full canopy. J. Amer. Soc. Hort. Sci. 125:93-99.

Zanotelli, D., M. Rechenmacher, W. Guerra, A Cassar, and M. Tagliavini. 2014. Seasonal uptake rate dynamics and partitioning of mineral nutrients by bourse shoots of field grown apple trees. Europ. J. of Hort. Sci. 79:203-211. 